Journal of Semantics 26: 367-392

doi:10.1093/jos/ffp008

Advance Access publication July 27, 2009

\title{
Branching Quantification v. Two-way Quantification
}

\author{
NINA GIERASIMCZUK AND JAKUB SZYMANIK \\ University of Amsterdam
}

\section{Abstract}

We discuss the thesis formulated by Hintikka (1973) that certain natural language sentences require non-linear quantification to express their meaning. We investigate sentences with combinations of quantifiers similar to Hintikka's examples and propose a novel alternative reading expressible by linear formulae. This interpretation is based on linguistic and logical observations. We report on our experiments showing that people tend to interpret sentences similar to Hintikka sentence in a way consistent with our interpretation.

\section{HINTIKKA'S THESIS}

Hintikka (1973) claims that the following sentences essentially require non-linear quantification for expressing their meaning.

(1) Some relative of each villager and some relative of each townsman hate each other.

(2) Some book by every author is referred to in some essay by every critic.

(3) Every writer likes a book of his almost as much as every critic dislikes some book he has reviewed.

Throughout the paper, we will refer to sentence (1) as Hintikka sentence. According to Hintikka, the interpretation of sentence (1) can be only expressed using Henkin's quantifier as follows:

$$
\left(\begin{array}{c}
\forall x \exists y \\
\forall z \exists w
\end{array}\right)((V(x) \wedge T(z)) \rightarrow(R(x, y) \wedge R(z, w) \wedge H(y, w)))
$$

where unary predicates $V$ and $T$ denote the set of villagers and the set of townsmen, respectively. The binary predicate symbol $R(x, y)$ denotes that the relation ' $x$ and $y$ are relatives' and $H(x, y)$ the symmetric relation ' $x$ and $y$ hate each other'.

Branching quantification (also called partially ordered quantification, Henkin quantification) was proposed by Henkin (1961) (for a survey, see Krynicki \& Mostowski 1995). Informally speaking, the idea of such constructions is that for different rows of quantifiers in a prefix, the 
values of the quantified variables are chosen independently. According to Henkin's semantics for branching quantifiers, formula (4) is equivalent to the following existential second-order sentence:

$$
\begin{aligned}
\exists f \exists g \forall x \forall z((V(x) \wedge T(z)) \rightarrow & (R(x, f(x)) \wedge R(z, g(z)) \\
& \wedge H(f(x), g(z)))) .
\end{aligned}
$$

Functions $f$ and $g$ (so-called Skolem functions) choose relatives for every villager and every townsman, respectively. Notice that the value of $f(g)$ is determined only by the choice of a certain villager (townsman). In other words, to satisfy the formula, relatives have to be chosen independently. ${ }^{1}$ This second-order formula is equivalent to the following sentence with quantification over sets:

$$
\begin{aligned}
& \exists A \exists B \forall x \forall z((V(x) \wedge T(z)) \rightarrow(\exists y \in A R(x, y) \\
& \wedge \exists w \in B R(z, w) \wedge \forall y \in A \forall w \in B H(\gamma, w))) .
\end{aligned}
$$

The existential second-order sentence is not equivalent to any first-order sentence (see the Barwise-Kunen theorem in Barwise 1979). Not only universal and existential quantifiers can be branched; the procedure of branching works in a very similar way for other quantifiers. Some examples are discussed in the next section of this paper.

The reading of Hintikka sentence given by formula (4) is called the branching reading. However, it can also be assigned weaker readings, that is linear representations which are expressible in elementary logic. Let us consider the following candidates:

$$
\text { (5) } \begin{aligned}
& \forall x \exists y \forall z \exists w((V(x) \wedge T(z)) \rightarrow(R(x, y) \wedge R(z, w) \wedge H(\gamma, w))) \\
& \wedge \forall z \exists w \forall x \exists y((V(x) \wedge T(z)) \rightarrow(R(x, y) \wedge R(z, w) \wedge H(y, w))) . \\
& \text { (6) } \quad \forall x \exists y \forall z \exists w((V(x) \wedge T(z)) \rightarrow(R(x, y) \wedge R(z, w) \wedge H(\gamma, w))) . \\
& \text { (7) } \quad \forall x \forall z \exists y \exists w((V(x) \wedge T(z)) \rightarrow(R(x, y) \wedge R(z, w) \wedge H(\gamma, w))) .
\end{aligned}
$$

In all these formulae, the choice of the second relative depends on the one that has been previously selected. To see the difference between the above readings and the branching reading, consider the second-order formula equivalent to the sentence (6):

$$
\begin{aligned}
\exists f \exists g \forall x \forall z((V(x) \wedge T(z)) \rightarrow & (R(x, f(x)) \wedge R(z, g(x, z)) \\
& \wedge H(f(x), g(x, z)))) .
\end{aligned}
$$

\footnotetext{
${ }^{1}$ The idea of branching is more visible in the case of simpler quantifier prefixes, like in sentence (8) discussed in section 3.2.
} 
It is enough to compare the choice functions in this formula with those in existential second-order formula corresponding to the sentence (4) to see the difference in the structure of dependencies required in both readings. Of course, dependencies in sentences (5) and (7) are analogous to (6). As a result, all the weaker readings are implied by the branching reading (4) (where both relatives have to be chosen independently). Therefore, we sometimes refer to the branching reading as the strong reading.

Formulae (5)-(7) are also ordered according to the inference relation which occurs between them. Obviously, formula (5) implies formula (6), which implies formula (7). Therefore, formula (5) is the strongest among the weak readings.

By Hintikka's thesis, we mean the following statement:

Hintikka's thesis. Hintikka sentences don't have an adequate linear reading. They should be assigned the strong reading and not any of the weaker readings.

Because of its many philosophical and linguistic consequences Hintikka's claim has sparked lively controversy (see e.g. Jackendoff 1972; Gabbay \& Moravcsik 1974; Guenthner \& Hoepelman 1976; Hintikka 1976; Stenius 1976; Barwise 1979; Bellert 1989; May 1989; Sher 1990; Mostowski 1994; Liu 1996; Beghelli et al. 1997; Janssen 2003; Mostowski \& Wojtyniak 2004; Szymanik 2005; Schlenker 2006; Gierasimczuk \& Szymanik 2007). In relation to that, there has also been a vivid discussion on the ambiguity of sentences with multiple quantifiers (see Kempson \& Cormack 1981a, 1981b, 1982; Tennant 1981; Bach 1982; May 1985; Jaszczolt 2002; Bott \& Radó 2009; Robaldo 2009).

In the present article, some of the arguments presented in the discussion are analysed and critically discussed. We propose to interpret Hintikka sentence by the first-order formula (5):

$$
\begin{aligned}
& \forall x \exists y \forall z \exists w((V(x) \wedge T(z)) \rightarrow(R(x, y) \wedge R(z, w) \wedge H(y, w))) \\
& \wedge \forall z \exists w \forall x \exists y((V(x) \wedge T(z)) \rightarrow(R(x, y) \wedge R(z, w) \wedge H(y, w))) .
\end{aligned}
$$

In the rest of this paper, we will refer to this reading as the two-way reading of Hintikka sentence, as opposed to one-way reading expressed by formula (6).

Our proposal turns out to agree with speakers' intuitions, as we show in the next section, and it is also consistent with speakers' behaviour. The latter fact is supported by empirical data, which we 
present in section $4 .^{2}$ Our main conclusion is that sentences with multiple quantifiers, including the Hintikka sentence, allow for a linear reading. This of course clearly contradicts Hintikka's thesis.

\section{MULTIQUANTIFIER SENTENCES}

Every branching quantifier can be expressed by some single generalized quantifier, so in the sense of definability Hintikka's thesis cannot be right. However, the syntax of branching quantification has a particular simplicity and elegance that is lost when translated into the language of generalized quantifiers. The procedure of branching does not employ new quantifiers. Instead, it enriches the syntactic means of arranging existing quantifiers, at the same time increasing their expressive power. Therefore, the general question is as follows: Are there sentences with simple determiners such that non-linear combinations of quantifiers corresponding to the determiners are essential to account for the meanings of those sentences? The affirmative answer to this question, suggested by Hintikka, claims existence of sentences with quantified noun phrases which are always interpreted scope independently. We show that for sentences similar to those proposed by Hintikka, the claim is not true.

Before we move on to the central problem, let us consider more sentences with combinations of at least two determiners. We are mainly interested in sentences whose branching interpretation is not equivalent to any linear reading. They all fall into the scope of our discussion and we will call them all 'Hintikka sentences'.

Interesting examples of Hintikka sentences, which we will discuss later, were given by Barwise (1979).

(8) Most villagers and most townsmen hate each other.

(9) One-third of the villagers and half of the townsmen hate each other.

These sentences seem to be more frequent in our everyday language and more natural than Hintikka's own examples, even though their adequate meaning representation is no less controversial.

\footnotetext{
${ }^{2}$ It is worth noticing that our proposal is reminiscent of the linguistic representation of reciprocals. For example, according to the seminal paper on 'each other' by Heim et al. (1991), Hintikka sentence has the following structure: $\mathrm{EACH}[[\mathrm{QP}$ and $\mathrm{QP}]][\mathrm{V}$ the other], where 'each' quantifies over the two conjuncts, which turns the sentence into [QP1 V the other and QP2 V the other], where 'the other' picks up the rest of quantifiers anaphorically. This interpretation is similar to the two-way reading.
} 
Many more examples have been given to justify the existence of non-linear semantic structures in natural language [see e.g. sentences (10)-(12)].

(10) I told many of the men three of the stories. (Jackendoff 1972)

(11) A majority of the students read two of those books. (Liu 1996)

(12) We have been fighting for many years for human rights in China. I recount the story of our failures and successes and say: 'Whenever a representative from each country fought for the release of at least one dissident from each prison, our campaign was a success'. (Schlenker 2006)

\section{THEORETICAL DISCUSSION OF HINTIKKA'S THESIS}

\subsection{A remark on possible readings}

Let us start with the following remark. It was observed by Mostowski (1994) that from Hintikka sentence (1), we can infer that

(13) Each villager has a relative.

This sentence obviously has the following reading: $\forall x(V(x) \rightarrow \exists y R(x, y))$. It can be false in a model with an empty town, if there is a villager without a relative. However, the strong reading of Hintikka sentence [see formula (1)], which has the form of an implication with a universally quantified antecedent, is true in every model with an empty town. Hence, the reading of (13) is not logically implied by proposed readings of Hintikka sentence. Therefore, the branching meaning of Hintikka sentence should be corrected to the following formula with restricted quantifiers:

$$
\begin{aligned}
& (\forall x: V(x))(\exists y: R(x, y)) \\
& (\forall z: T(z))(\exists w: R(z, w))
\end{aligned}
$$

which is equivalent to

$$
\begin{aligned}
& \exists A \exists B(\forall x(V(x) \rightarrow \exists y \in A R(x, y)) \\
& \wedge \forall z(T(z) \rightarrow \exists w \in B R(z, w)) \wedge \forall y \in A \forall w \in B H(y, w)) .
\end{aligned}
$$

Observe that similar reasoning can be used to argue for restricting quantifiers in formulae expressing different possible meanings of all our sentences. However, applying these corrections uniformly would not change the main point of our discussion. We still would have to choose between the same number of possible readings, the only difference being the restricted quantifiers. Therefore, for simplicity, we will 
forego these corrections. From now on, we will assume that all predicates in our formulae have non-empty denotation.

\subsection{Hintikka sentences are symmetric}

It has been observed that there is a strong intuition that the two following versions of Hintikka sentence are equivalent (Hintikka 1973):

(1) Some relative of each villager and some relative of each townsman hate each other.

(15) Some relative of each townsman and some relative of each villager hate each other.

However, if we assume that formula (6) repeated here

(6) $\forall x \exists y \forall z \exists w((V(x) \wedge T(z)) \rightarrow(R(x, y) \wedge R(z, w) \wedge H(y, w)))$

is an adequate reading of sentence (1), then we also have to assume that an adequate reading of sentence (15) is represented by the formula:

$$
\forall z \exists w \forall x \exists y((V(x) \wedge T(z)) \rightarrow(R(x, y) \wedge R(z, w) \wedge H(y, w))) .
$$

However, (6) and (16) are not logically equivalent and therefore, it would be wrong to treat them as correct interpretations of sentences (1) or (15). Therefore, we have to reject readings (6) and (16) from the set of possible alternatives.

Notice that a similar argument applies when we consider other Hintikka sentences. For instance, it is enough to observe that the following sentences are also equivalent:

(8) Most villagers and most townsmen hate each other.

(17) Most townsmen and most villagers hate each other.

However, the possible one-way linear reading of (8)

(18) $\operatorname{MOST} x(V(x), \operatorname{MOST} y(T(y), H(x, y)))$

is not equivalent to an analogous reading of (17). Hence, the one-way linear reading in (18) cannot be right.

One of the empirical tests we conducted was aimed at checking whether people really consider pairs like (8) and (17) to be equivalent. The results that we will present prove that this is the case. Therefore, the argument from symmetry is also cognitively convincing (see section 4.4.1 for a description of the experiment and section 4.4.2 for our empirical results). Despite this observation, we cannot conclude the 
validity of Hintikka's thesis so easily. First, we have to consider the remaining weak candidates, that is formulae (5) and (7):

$$
\begin{aligned}
& \forall x \exists y \forall z \exists w((V(x) \wedge T(z)) \rightarrow(R(x, y) \wedge R(z, w) \wedge H(\gamma, w))) \\
& \wedge \forall z \exists w \forall x \exists y((V(x) \wedge T(z)) \rightarrow(R(x, y) \wedge R(z, w) \wedge H(\gamma, w))), \\
& \forall x \forall z \exists y \exists w((V(x) \wedge T(z)) \rightarrow(R(x, y) \wedge R(z, w) \wedge H(\gamma, w))) .
\end{aligned}
$$

Hintikka does not consider either of these, and other authors focus only on formula (7) (see e.g. Barwise 1979; Mostowski \& Wojtyniak 2004). Also for different Hintikka sentences, we still have to differentiate between some possibilities. As an alternative for formula (18), we can consider not only the branching reading (19) [equivalent to (20)]

$$
\left(\begin{array}{l}
\operatorname{MOST} x: V(x) \\
\operatorname{MOST} y: T(y)
\end{array}\right) H(x, y)
$$

$$
\begin{aligned}
& \exists A \exists B(\operatorname{MOST} x(V(x), A(x)) \wedge \operatorname{MOST} y(T(x), B(y)) \wedge \\
& \forall x \in A \forall y \in B H(x, y))
\end{aligned}
$$

but also the two-way meaning

$$
\begin{aligned}
& \operatorname{MOST} x(V(x), \operatorname{MOST} y(T(y), H(x, y))) \\
& \wedge \operatorname{MOST} y(T(y), \operatorname{MOST} x(V(x), H(y, x))) .
\end{aligned}
$$

Notice that for proportional sentences, like (8), there is no interpretation corresponding to the weakest reading of Hintikka sentence, formula (7), as proportional sentences contain only two simple determiners and not four as in Hintikka's original example. This observation already indicates that the two-way form, as a uniform representation of all Hintikka sentences, should be preferred to the weakest reading. We will present a further argument against the weakest reading (7) in the next section.

To sum up, the symmetry argument rules out readings with asymmetric scope dependencies. At this point, the adequacy of the weakest reading is also controversial since it is not uniform: it cannot be extended to proportional sentences. Our space of possibilities now consists of the branching and the two-way reading. In the next section, we give further reasons to reject the weakest reading of Hintikka's sentence.

\subsection{Inferential arguments}

Let us now move on to Mostowski's (1994) argument against the weakest reading of Hintikka sentences. Consider the following: 
Some relative of each villager and some relative of each townsman hate each other.

Mark is a villager.

Therefore: Some relative of Mark and some relative of each townsman hate each other.

In other words, if we assume that Mark is a villager, then we have to agree that Hintikka sentence implies that some relative of Mark and some relative of each townsman hate each other.

If we interpret Hintikka sentence as having the weakest meaning (7)

$$
\forall x \forall z \exists y \exists w((V(x) \wedge T(z)) \rightarrow(R(x, y) \wedge R(z, w) \wedge H(\gamma, w))),
$$

then we have to agree that the following sentence is true in Figure 1.

(1) Some relative of Mark and some relative of each townsman hate each other.

Mostowski (1994) observes that this is a dubious consequence of the weakest reading. He claims that sentence (1) intuitively has the following reading:

(2) $\exists x(R($ Mark, $x) \wedge \forall y(T(\gamma) \rightarrow \exists z(R(\gamma, z) \wedge H(x, z))))$.

Formula (2) is false in the model of Figure 1. Therefore, it cannot be implied by the weakest reading of Hintikka sentence which is true in the model. However, it is implied by the strong reading which is also false in the model. Hence, Mostowski concludes that Hintikka sentence cannot have the weakest reading (7).

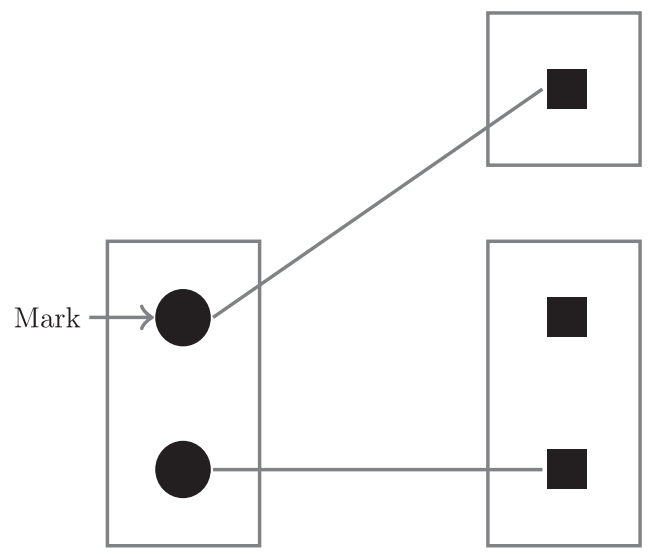

Figure 1 Relatives of Mark are on the left; on the right are two town families. 
If Mostowski's intuition is correct (which could be established by experimental means), then we can conclude from this argument that the weakest reading, (7), should be eliminated from the set of possible alternatives. Then we are left with two propositions: the branching and the two-way interpretation. Both of them have the desired inference properties.

3.3.1 Negation normality. In his paper on Hintikka's thesis, Barwise (1979) refers to the notion of negation normality in a defence of the statement that the proper interpretation of Hintikka sentence is an elementary formula. He observes that negations of some simple quantifier sentences, that is sentences without sentential connectives other than 'not' before a verb, can easily be formulated as simple quantifier sentences. In some cases, this is impossible. Namely, the only way to negate some simple sentences is by prefixing them with the phrase 'it is not the case that' or an equivalent expression of a theoretical character. Sentences of the first kind are called negation normal. For example, sentence

(3) Everyone owns a car.

can be negated normally as follows:

(4) Someone doesn't own a car.

As an example of a statement which is not negation normal consider the following (see Barwise 1979):

(5) The richer the country, the more powerful its ruler.

It seems that the most efficient way to negate it is as follows:

(6) It is not the case that the richer the country, the more powerful its ruler.

Barwise proposes to treat negation normality as a test for first-order definability with respect to sentences with combinations of elementary quantifiers. This proposal is based on the following theorem.

Theorem 1. If $\phi$ is a sentence definable in $\sum_{1}^{1}$, the existential fragment of second-order logic, and its negation is logically equivalent to a $\sum_{1}^{1}$-sentence, then $\phi$ is logically equivalent to some first-order sentence.

Barwise claims that the results of the negation normality test suggest that people tend to find Hintikka sentence to be negation normal, and hence definable in elementary logic. According to Barwise, people 
tend to agree that the negation of Hintikka sentence can be formulated as follows:

(7) There is a villager and a townsmen that have no relatives that hate each other.

Barwise's claim excludes the branching reading of Hintikka sentence but is consistent with the two-way interpretation. Therefore, in case of Hintikka sentence, we are left with only one possible reading: the twoway reading. However, Barwise's argument does not apply to the proportional sentences, as proportional quantifiers are not definable in first-order logic. Therefore, in the case of proportional sentences, we still have to choose between the branching and the two-way interpretation.

\subsection{Complexity arguments}

Mostowski \& Wojtyniak (2004) claim that native speakers' inclination towards a first-order reading of Hintikka sentence can be explained by means of computational complexity theory (see e.g. Papadimitriou 1993). The authors prove that the problem of recognizing the truth value of the branching reading of Hintikka sentence in finite models is an NPTIME-complete problem. ${ }^{3}$ It can also be shown that proportional branching sentences define an NPTIME-complete class of finite models (see Sevenster 2006).

Assuming that the class of practically computable problems is identical with the PTIME class (i.e. the tractable version of ChurchTuring thesis; see Edmonds 1965), it may be argued that the human mind is not equipped with mechanisms for recognizing NPTIMEcomplete problems. ${ }^{4}$ In other words, in many situations, an algorithm for checking the truth value of the strong reading of Hintikka sentence is intractable. According to Mostowski \& Wojtyniak (2004), native speakers can only choose between meanings which are practically computable. The two-way reading is PTIME computable ${ }^{5}$ and therefore, even taking into account computational restrictions, is more plausible than the branching reading.

\footnotetext{
${ }^{3}$ NPTIME-complete problems are computationally the most difficult problems in the NPTIME class. In particular, PTIME = NPTIME if any NPTIME-complete problem is PTIME computable. PTIME (NPTIME) is the class of problems which can be solved by a (non-deterministic) Turing machine in a number of steps bounded by a polynomial function of the length of a query. See Garey \& Johnson (1979) for more details.

${ }_{4}$ This statement can be given independent psychological support (see e.g. Frixione 2001).

${ }^{5}$ As model checking for first-order sentences is PTIME computable (see e.g. Immerman 1998).
} 


\subsection{Conclusions}

In the foregoing, we discussed possible obstacles to various interpretations of Hintikka sentences. Our two-way reading for Hintikka sentences is the only reading satisfying all the following properties:

- It is symmetric.

- It ensures a uniform reading for all Hintikka sentences.

- It passes Mostowski's inferential test.

- It is negation normal for Hintikka sentence.

- Its truth value is practically computable in finite models.

In the next section, we will present empirical arguments that the twoway reading is consistent with the interpretation people most often assign to Hintikka sentences.

\section{EMPIRICAL EVIDENCE FOR THE TWO-WAY READING}

Many of the authors taking part in the dispute on the proper logical interpretation of Hintikka sentences have argued not only from their own linguistic intuitions but also from the universal agreement of native speakers. For instance, Barwise claims that

In our experience, there is almost universal agreement rejecting Hintikka's claim for a branching reading (Barwise 1979).

However, none of these authors have provided genuine empirical data to support their claims. In the rest of this section, we present experimental work supporting the two-way reading.

\subsection{Experimental hypotheses}

Our hypotheses are as follows.

Hypothesis 1. People treat Hintikka sentences as symmetric sentences.

This was theoretically justified by Hintikka (1973) and discussed in section 3.2. To be more precise, we predict that subjects will treat sentences like (8) and (9) as equivalent.

(8) More than 3 villagers and more than 5 townsmen hate each other.

(9) More than 5 townsmen and more than 3 villagers hate each other. 
Hypothesis 2. In an experimental context, the preferred reading of the Hintikka sentences is best represented by the two-way formula.

Based on the arguments of the last section, we predict that subjects will tend to assign the two-way reading to Hintikka sentences, that is they will accept Hintikka sentence when confronted with a model that satisfies its two-way interpretation. We also predict that the comprehension of Hintikka sentences is similar in English and Polish-in both languages native speakers accept the two-way reading.

\subsection{Subjects}

Subjects were native speakers of English and native speakers of Polish who volunteered to take part in the experiment. They were undergraduate students in computer science at Stanford University and in philosophy at Warsaw University. All subjects had elementary training in logic so that they could understand the instructions. The experiment was conducted with 32 computer science students and 90 philosophy students.

\subsection{Materials}

It was suggested by Barwise \& Cooper (1981) and empirically verified by Geurts \& van der Slik (2005) (see also Szymanik 2009) that the monotonicity of quantifiers influences how difficult they are to comprehend. In particular, sentences containing downward monotone quantifiers are more difficult to reason with than sentences containing only upward monotone quantifiers. ${ }^{6}$ For this reason, in the experiment, we only used (combinations of) monotone increasing quantifiers of the form 'More than $n$ ' in otherwise simple sentences.

In our tasks, the quantifiers referred to shape of geometrical objects (circles and squares). The sentences were Hintikka sentences (e.g. see sections 4.4 .1 and 4.4.3).

\subsection{Experiments}

The study was conducted in two languages and consisted of two parts. It was a paper-and-pencil study. There were no time limits and it took 20 minutes on average for all students to finish the test. Below we present descriptions of each part of the English version (Appendix A) of the test. The Polish test was analogous.

\footnotetext{
${ }^{6}$ A quantifier $Q_{M}$ is upward monotone (increasing) if the following holds: if $Q_{M}(A)$ and moreover $A \subseteq B \subseteq M$, then $Q_{M}(B)$. The downward monotone (decreasing) quantifiers are defined analogously as being closed on taking subsets.
} 
4.4.1 Experiment I: are Hintikka sentences symmetric? The first part of the test was designed to check whether subjects treat Hintikka sentences as symmetric (see section 3.2 for a discussion). Recall the notion of symmetry for our sentences. Let $Q_{1}, Q_{2}$ be quantifiers and $\psi$ a quantifier-free formula. We will say that sentence $Q_{1} x Q_{2} \gamma \psi(x, y)$ is symmetric if and only if it is equivalent to $Q_{2} y Q_{1} x \psi(x, y)$. In other words, switching the whole-quantifier prefix (determiner + noun phrase) does not change its meaning.

In order to check whether subjects treat sentences with switched quantifier prefixes as equivalent, we presented them with sentence pairs $\phi, \phi^{\prime}$ and asked whether the first sentence implies the second sentence. There were 20 tasks. Ten of them were valid inference patterns provided symmetry holds. The rest were fillers, six of which were invalid patterns similar to the symmetric case. In three of these, we changed the order of nouns, that is we had $Q_{1} x Q_{2} \gamma \psi(x, y)$ and $Q_{1} y$ $Q_{2} x \psi(x, y)$. In the remaining three, we switched determiners (rather than complete quantifier phrases), that is $Q_{1} x Q_{2} \gamma \psi(x, y)$ and $Q_{2} x Q_{1} y$ $\psi(x, y)$. Four of the tasks were simple valid and invalid inferences with the quantifiers 'more than', 'all' and 'some'.

We constructed our sentences using non-existing nouns to eliminate pragmatic influence on subjects' answers. For example, in the English version (Appendix) of the test, we used nouns proposed by Soja et al. (1991): mells, stads, blickets, frobs, wozzles, fleems, coodles, doffs, tannins, fitches and tulvers. In Polish, we had the following nouns: strzew, memniak, balbasz, protorożec, melarek, krętowiec, stular, wachlacz, fisut, bubrak and wypsztyk. Our subjects were informed that they were not supposed to know the meanings of the common nouns occurring in the sentences. Figure 2 gives examples of each type of task in English.

We excluded the possibility of interpreting the sentences as being about the relations between objects of the same kind (e.g. '68 coodles hate each other') by explicitly telling the subjects that in this setting the relation can occur only between objects from two different groups.

4.4.2 Results. Our main finding was that $94 \%\left[\chi^{2}=709.33\right.$, degrees of freedom $(\mathrm{df})=1, P<0.001]$ and $98 \%\left(\chi^{2}=286.90\right.$, df $=1, P<$ 0.001 ) of the responses were in agreement with our symmetry hypothesis in the group consisting of philosophy undergraduates at Warsaw University and among Stanford University computer science students, respectively. Moreover, for the sake of completeness, we report results for the remaining part of the experiment. In simple inferences, $83 \%\left(\chi^{2}=153.4, \mathrm{df}=1, P<0.001\right)$ and $97 \%\left(\chi^{2}=110.63\right.$, 


More than 12 fleems and more than 13 coodles hate each other.
More than 13 coodles and more than 12 fleems hate each other.
VALID

More than 20 wozzles and more than 35 fitches hate each other.

More than 20 fitches and more than 35 wozzles hate each other.

VALID NOT VALID

More than 105 wozzles and more than 68 coodles hate each other.

More than 68 wozzles and more than 105 coodles hate each other.

VALID NOT VALID

Some tulvers are mells.
VALID
Some mells are tulvers.
NOT VALID

Figure 2 Four tasks from the first experiment: symmetry pattern, two invalid patterns and simple inference.

$\mathrm{df}=1, P<0.001)$ of the answers were logically correct. For invalid symmetry inferences, the results were $86 \%\left(\chi^{2}=286.02\right.$, df $=1, P<$ $0.001)$ and $93 \%\left(\chi^{2}=138.38, \mathrm{df}=1, P<0.001\right)$ (see Figure 3$)$. This is a statistically significant result for both groups. ${ }^{7}$ Therefore, our first hypothesis - that people treat Hintikka sentences as symmetric-was confirmed.

We also compared the performance of the two groups (philosophers v. computer scientists) with respect to the three kinds of tests and found no statistically significant differences. To be more precise, there was no difference either in the symmetry task $\left(\chi^{2}=6.583\right.$, $\left.\mathrm{df}=6, P=0.361\right)$, in the simple inferences $\left(\chi^{2}=8.214\right.$, $\left.\mathrm{df}=4, P=0.084\right)$, or in the invalid arguments $\left(\chi^{2}=3.888, \mathrm{df}=4, P=0.421\right)$.

\footnotetext{
${ }^{7}$ We were only interested in the frequency of correct answers among all answers to the tasks based on the valid symmetric inference pattern (simple inferences and inferences based on the logically invalid schema were treated as fillers) and that is why we used $\chi^{2}$ to analyse our data and not a statistical model, like multivariate analysis of variance (MANOVA), in which the observed variance is partitioned into components due to different independent (explanatory) variables (e.g. two groups of subjects, four types of tasks). We did not analyse the data with MANOVA because the following assumptions were violated (see e.g. Ferguson \& Takane 1990): According to our hypothesis, we had expected that the number of answers 'valid' will dominate. In other words, the normality assumption of MANOVA was not satisfied, that is the distribution of the answers is not normal but skewed $(-4.728)$ towards validity, which was a further reason for using a non-parametric test. Additionally, the conditions (within subject) for each kind of tasks were different (the number of problems varied between 10, 4, 3 and 3) and the groups were not equal (90 philosophers, 32 computer scientists), which also indicates the use of non-parametric statistical model.
} 


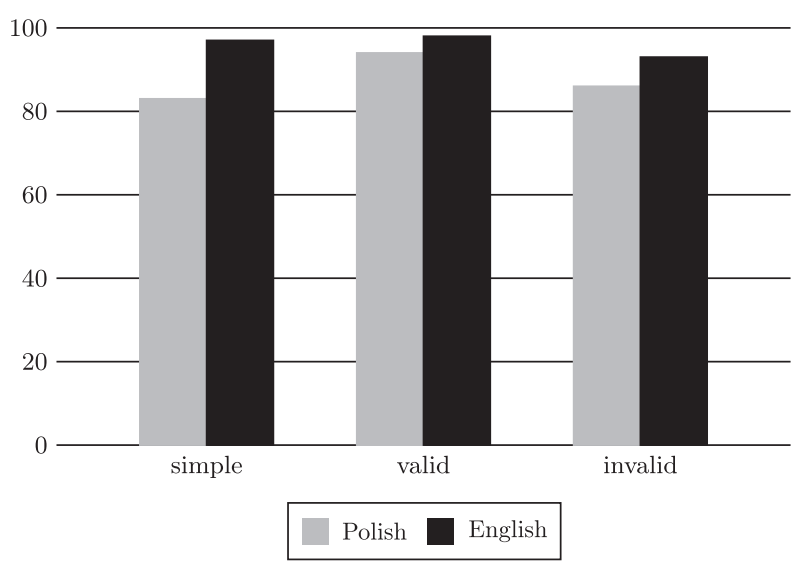

Figure 3 Percentage of correct answers in the first test.

4.4.3 Experiment II: branching v. two-way interpretation. The second questionnaire was the main part of the experiment, designed to discover whether people agree with the two-way reading of Hintikka sentences. Subjects were presented with nine non-equivalent Hintikka sentences. Every sentence was paired with a model. All but two sentences were accompanied by a picture satisfying the two-way reading but not the branching reading. The remaining control tasks consisted of pictures in which the associated sentences were false, regardless of which of the possible interpretations was chosen. ${ }^{8}$ Every illustration was black and white and showed irregularly distributed squares and circles. Some objects of different shapes were connected with each other by lines. The number of objects in the pictures varied between 9 and 13 and the number of lines was between 3 and 15 .

All critical sentences were of the following form, where $1 \leqslant n, m \leqslant 3$ :

(10) More than $n$ squares and more than $m$ circles are connected by lines.

(11) Więcej niż $n$ kwadraty i więcej niż $m$ koła są połączone liniami.

Notice that some Hintikka sentences contain the phrase 'each other'. However, we decided not to use this phrase in the sentences tested in the main part of the experiments. This was because our previous experiments (Gierasimczuk \& Szymanik 2007) indicated that the occurrence of reciprocal expressions in these sentences made people

\footnotetext{
${ }^{8}$ Bott \& Radó (2007) empirically assessed this methodology for studying quantifier scope and demonstrated its reliability and validity.
} 
More than 1 square and more than 2 circles are connected by lines.

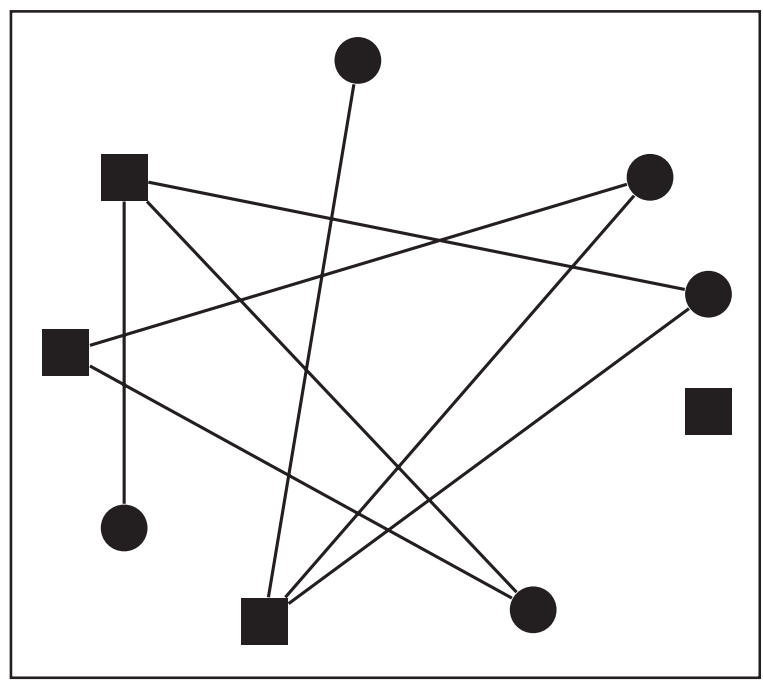

TRUE

FALSE

Figure 4 Two-way task from the second part of the experiment.

interpret them as statements about the existence of lines between figures of the same geometrical shape, which is not the interpretation we wanted to test.

In the first test, the usage of the phrase 'each other' made the 'hating' relation symmetric. In this experiment, the relation 'being connected by a line' is already symmetric in itself. Moreover, interviews with native speakers suggest that in the context of the relation 'being connected by lines' omitting 'each other' leads to more natural sentences. Additionally, in the Polish version of the sentences, there is no grammatically possible phrase corresponding to 'each other'.

Figures 4 and 5 show two examples of our tasks. In the first picture, the two-way reading is true and the branching reading is false. In the second picture, the sentence is false on either readings. The subjects were asked to decide if the sentence is a true description of the picture.

4.4.4 Results. We got the following results ${ }^{9}: 94 \%\left(\chi^{2}=444.19\right.$, df $=$ $1, P<0.001)$ of the answers of the philosophy students and $96 \%\left(\chi^{2}=\right.$ 187.61, $\mathrm{df}=1, P<0.001)$ of the answers of the computer science students were two-way, that is 'true' when the picture represented

\footnotetext{
${ }^{9}$ We used a non-parametric statistical test for the same reasons as in the first experiment.
} 
More than 3 circles and more than 2 squares are connected by lines.

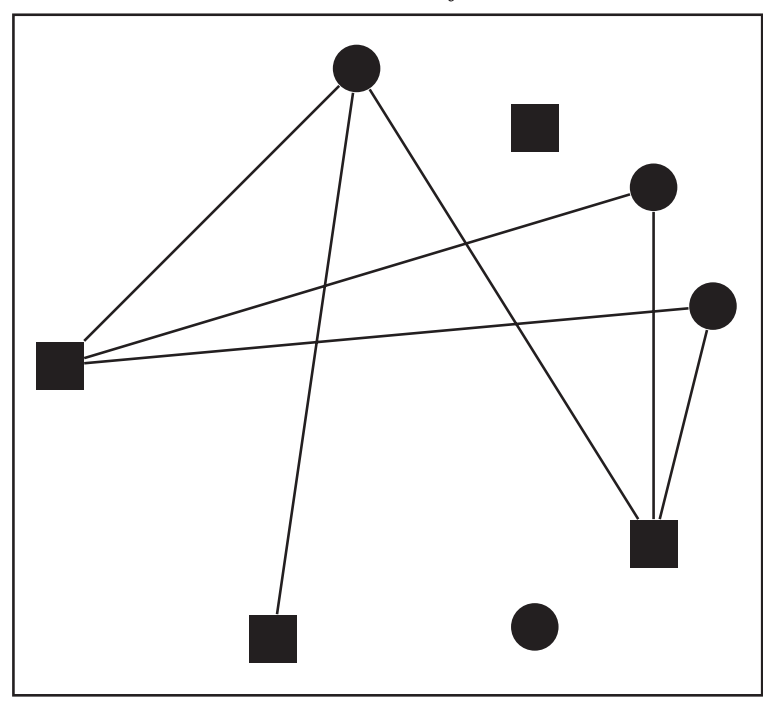

TRUE

FALSE

Figure 5 An example of a false task from the second part of the experiment.

a model for a two-way reading of the sentence. For the two sentences that were false, no matter how subjects interpreted them, the rates of correct answers were $92 \%\left(\chi^{2}=136.94, \mathrm{df}=1, P<0.001\right)$ and $96 \%$ $\left(\chi^{2}=50.77, \mathrm{df}=1, P<0.001\right)$. These results are statistically significant. Therefore, our second hypothesis - that in an empirical context people can assign to Hintikka sentences meanings which are best represented by two-way formulae-was confirmed.

Further analysis of the individual subjects' preferences revealed that $94 \%$ of the philosophers $\left(\chi^{2}=71.11, P<0.001, \mathrm{df}=1\right)$ and $97 \%$ of the computer scientists $\left(\chi^{2}=28.12, P<0.001, \mathrm{df}=1\right)$ agreed on the two-way reading in more than half of the cases. Moreover, 67 $\left(74 \%, \chi^{2}=21.51, P<0.001, \mathrm{df}=1\right)$ philosophers and $28\left(88 \%, \chi^{2}=\right.$ $18, P<0.001, \mathrm{df}=1)$ computer scientists chose two-way readings in all tasks (see Table 1 for a presentation of all data).

Once again, we did not observe any differences between our two subject groups either in judging obviously false situations $\left(\chi^{2}=0.188\right.$, $\mathrm{df}=1, P=0.664)$ or in the two-way preferences $\left(\chi^{2}=3.900, \mathrm{df}=7\right.$, $P=0.791)$. Therefore, we conclude that with respect to the interpretation of quantifier combinations in Hintikka sentences there is no difference between English and Polish. 
384 Branching Quantification v. Two-way Quantification

\begin{tabular}{lcc} 
Groups & $\begin{array}{l}\text { Polish } \\
\text { philosophers }\end{array}$ & $\begin{array}{l}\text { American } \\
\text { computer scientists }\end{array}$ \\
\hline Number of subjects & 90 & 32 \\
Two-way answers & $94 \%$ & $95 \%$ \\
Recognized falsity & $92 \%$ & $96 \%$ \\
\hline
\end{tabular}

Table 1 Results of the second test

\section{CONCLUSIONS AND PERSPECTIVES}

\subsection{Conclusions}

Contrary to what Hintikka (1973) and many of his followers have claimed, we argue that Hintikka sentences have readings expressible by linear formulae that satisfy all conditions which prompted the introduction of branching interpretation. The reasons for treating such natural language sentences as having Fregean (linear) readings are twofold. In section 1, we discussed a number of theoretical arguments, which can be summed up as follows.

i. For Hintikka sentence, we should focus on four possibilities: a branching reading (4) and three weak readings: (5), (6) and (7).

ii. Hintikka's argument from symmetry given in section 3.2, together with the results of our first experiment, allows us to reject asymmetric formulae. A similar argument leads to rejecting the linear readings of other Hintikka sentences.

iii. What about the weakest reading? It does not exist for some Hintikka sentences so it cannot be viewed as a universal reading for all of them. Moreover, the inferential argument from section 3.3 suggests that the weakest meaning is also not an appropriate reading of Hintikka sentence.

iv. Therefore, there are only two alternatives: we have to choose between the two-way (5) and the branching readings (4).

In section 4, we discussed our empirical results. They indicate that people interpret Hintikka sentences in accordance with the two-way reading, at least in an experimental context. Additionally, we observed no statistically significant differences in preferences of native English and native Polish subjects.

Moreover, our experimental arguments are supported by the following observations. 
i. The argument by Barwise from negation normality, discussed in section 3.3.1, agrees with our empirical results.

ii. Branching readings, being NP-complete, may be too difficult for language users. Two-way readings, which are PTIME computable, are much easier in this sense.

Hence, even though we in principle agree that Hintikka sentences are ambiguous between all proposed readings, our experiments and theoretical considerations convince us that in some situations the proper reading of Hintikka sentences can be given by two-way formulae. This clearly contradicts Hintikka's thesis.

\subsection{Perspectives}

We have tested one of the best known among non-Fregean combinations of quantifiers, the so-called Hintikka sentences. We have presented arguments that these sentences can be interpreted in natural language by Fregean combinations of quantifiers. However, there is still some research to be done here. One can find and describe linguistic situations in which Hintikka sentences demand a branching analysis [recall example (12)]. For example, the work of Schlenker (2006) goes in this direction. Moreover, it is interesting to ask which determiners allow a branching interpretation at all (see e.g. Beghelli et al. 1997). Finally, we did not discuss the interplay of our proposition with the collective reading of noun phrases (see e.g. Lønning 1997) and various interpretations of reciprocal expressions (see Dalrymple et al. 1998).

As to the empirical work, we find a continuation towards covering other quantifier combinations exciting and challenging. Some ideas we discussed in the context of Hintikka sentences, such as inferential meaning, negation normality and the computational complexity perspective, seem universal and potentially useful for studying other quantifier combinations.

\section{Acknowledgements}

We would like to thank two anonymous reviewers of Journal of Semantics and Johan van Benthem, Tadeusz Ciecierski, Paul Dekker, Bogdan Dziobkowski, Bart Geurts, Justyna Grudzińska, Tikitu de Jager, Theo Janssen, Dick de Jongh, Allen Mann, Marcin Mostowski, Rick Nouwen, Eric Pacuit, Ingmar Visser, Yoad Winter, Łukasz Wojtyniak and Marcin Zajenkowski. The first author is a recipient of the 2009 Foundation for Polish Science Grant for Young Scientists. The second author was supported by a Marie Curie Early Stage Research fellowship in the project GLoRiClass (MEST-CT-2005-020841). 
386 Branching Quantification v. Two-way Quantification

NINA GIERASIMCZUK

Institute for Logic,

Language and Computation

Universiteit van Amsterdam

Science Park 904

1098 XH Amsterdam

The Netherlands

e-mail:n.gierasimczuk@uva.nl
JAKUB SZYMANIK

Institute for Logic,

Language and Computation

Universiteit van Amsterdam

Science Park 904

1098 XH Amsterdam

The Netherlands

e-mail:j.szymanik@uva.nl

\section{APPENDIX: ENGLISH VERSION OF THE TEST}

\section{A. First test}

Instruction: Over the next pages, you will find 20 tasks. Each task represents some inference. Your aim is to decide whether this inference is valid.

In other words, each task consists of two sentences with a horizontal line between them. You must decide whether a sentence above the line implies a sentence below the line.

If you think that inference pattern is valid (second sentence is implied by the first one) encircle: 'VALID', otherwise encircle: 'NOT VALID'.

\section{Example 1:}

At least 5 mells are stads.

At least 3 mells are stads.

\section{Example 2:}

\section{NOT VALID}

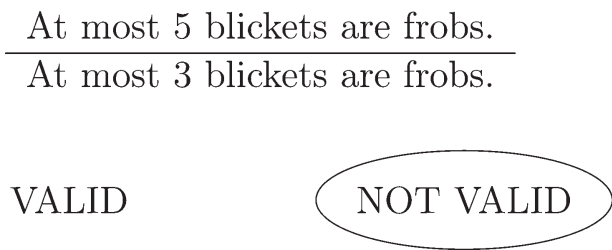

The following pairs of sentences were used in the test.

- More than 6 fleems are tulvers. More than 5 fleems are tulvers.

- More than 12 fleems and more than 13 coodles hate each other. More than 13 coodles and more than 12 fleems hate each other. 
- More than 16 stads and more than 9 blickets hate each other. More than 9 blickets and more than 16 stads hate each other.

- More than 16 mells and more than 25 blickets hate each other. More than 25 blickets and more than 16 mells hate each other.

- More than 10 mells are fleems. More than 11 mells are fleems.

- More than 9 frobs and more than 8 coodles hate each other. More than 8 coodles and more than 9 frobs hate each other.

- More than 20 wozzles and more than 35 fitches hate each other. More than 20 fitches and more than 35 wozzles hate each other.

- All wozzles are fleems. All fleems are wozzles.

- More than 100 wozzles and more than 150 stads hate each other. More than 150 stads and more than 100 wozzles hate each other.

- More than 105 wozzles and more than 68 coodles hate each other. More than 68 wozzles and more than 105 coodles hate each other.

- More than 6 doffs and more than 5 fitches hate each other. More than 5 fitches and more than 6 doffs hate each other.

- More than 47 stads and more than 55 tannins hate each other. More than 47 tannins and more than 55 stads hate each other.

- More than 58 frobs and more than 49 tannins hate each other. More than 49 frobs and more than 58 tannins hate each other.

- More than 7 coodles and more than 6 doffs hate each other. More than 6 doffs and more than 7 coodles hate each other.

- Some tulvers are mells. Some mells are tulvers.

- More than 99 coodles and more than 68 tulvers hate each other. More than 68 tulvers and more than 99 coodles hate each other.

- More than 7 tannins and more than 8 fitches hate each other. More than 8 fitches and more than 7 tannins hate each other.

- More than 19 frobs and more than 11 fleems hate each other. More than 11 fleems and more than 19 frobs hate each other.

- More than 159 stads and more than 25 fitches hate each other. More than 159 fitches and more than 25 stads hate each other.

- More than 8 frobs and more than 27 doffs hate each other. More than 27 frobs and more than 8 doffs hate each other. 


\section{B. Second test}

Instruction: Over the next few pages, you will find nine tasks to solve. Each task consists of a picture. Above every picture, there is exactly one sentence. Encircle TRUE if and only if the sentence is a true description of the picture. Otherwise, encircle FALSE.

More than 1 square and more than 2 circles are connected by lines.

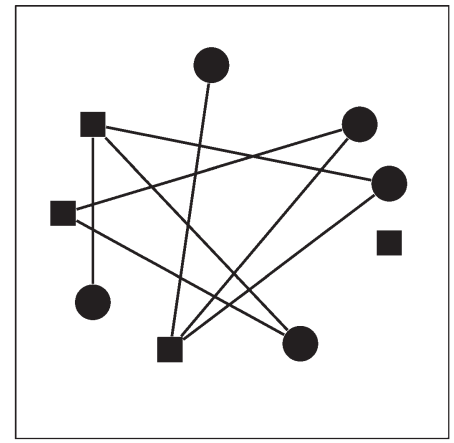

TRUE

FALSE

More than 3 circles and more than 2 squares are connected by lines.

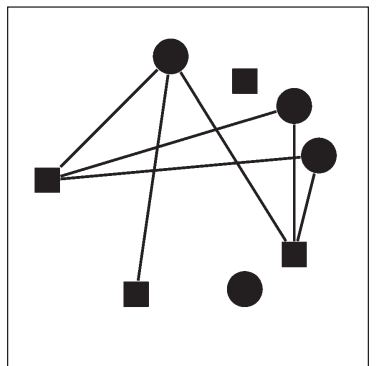

More than 1 square and more than 1 circle are connected by lines.

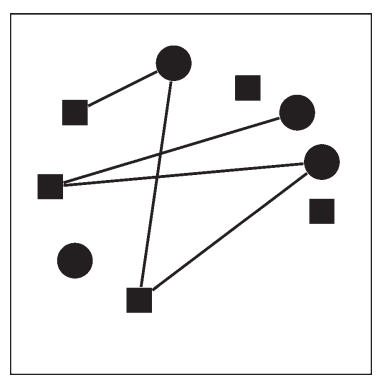


More than 3 circles and more than 1 square are connected by lines.

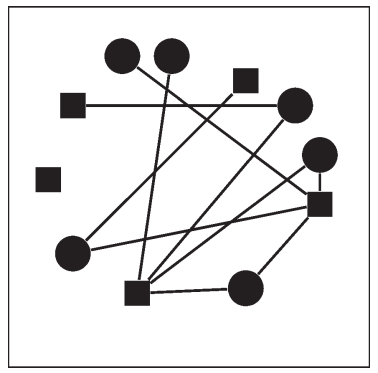

More than 3 circles and more than 3 squares are connected by lines.

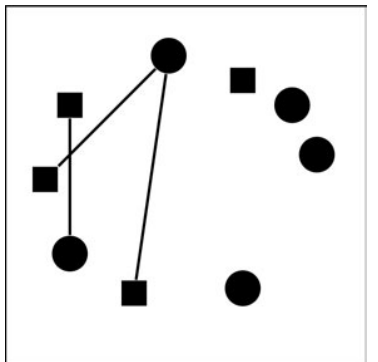

More than 2 circles and more than 3 squares are connected by lines.

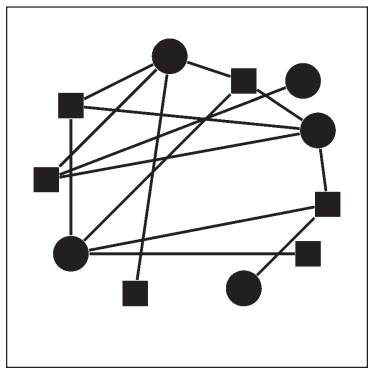

More than 3 circles and more than 3 squares are connected by lines.

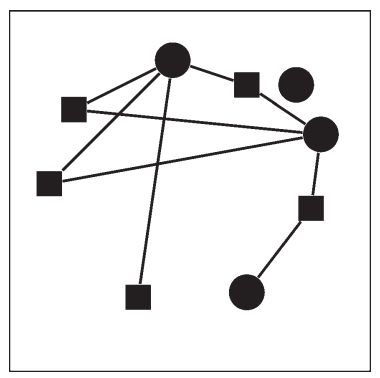


More than 2 squares and more than 1 circle are connected by lines.

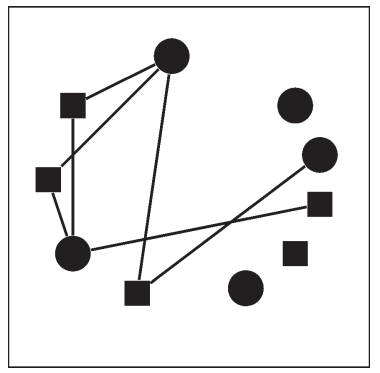

More than 2 squares and more than 1 circle are connected by lines.

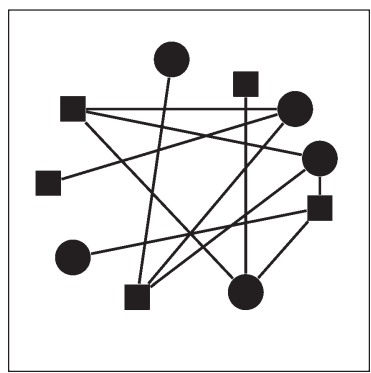

\section{REFERENCES}

Bach, K. (1982), 'Semantic nonspecificity and mixed quantifiers'. Linguistics and Philosophy 4:593-605.

Barwise, J. (1979), 'On branching quantifiers in English'. Journal of Philosophical Logic 8:47-80.

Barwise, J. \& Cooper, R. (1981), 'Generalized quantifiers and natural language'. Linguistics and Philosophy 4:159-219.

Beghelli, F., Ben-Shalom, D., \& Szabolcsi, A. (1997), 'Variation, distributivity, and the illusion of branching'. In A. Szabolcsi (ed.), Ways of Scope Taking, vol. 65. Studies in Linguistic and Philosophy. Kluwer Academic Publisher. The Netherlands. 29-69.

Bellert, I. (1989), Feature System for Quantification Structures in Natural
Language. Foris Publications, Dordrecht.

Bott, O. \& Radó, J. (2007), 'Quantifying quantifier scope: a cross-methodological comparison'. In S. Featherston \& W. Sternefeld (eds.), Roots-Linguistics in Search of Its Evidential Base, vol. 96. Studies in Generative Grammar. Mouton de Gruyter. Berlin. 53-74.

Bott, O. \& Radó, J. (2009), 'How to provide exactly one interpretation for every sentence, or what eye movements reveal about quantifier scope'. In S. Winkler \& S. Featherson (eds.), The fruits of empirical linguistics, vol. 1. Walter de Gruyter. Berlin.

Dalrymple, M., Kanazawa, M., Kim, Y., Mchombo, S., \& Peters, S. (1998), 'Reciprocal expressions and the 
concept of reciprocity'. Linguistics and Philosophy 21:159-210.

Edmonds, J. (1965), 'Paths, trees, and flowers'. Canadian Journal of Mathematics 17:449-467.

Ferguson, G. A. \& Takane, Y. (1990), Statistical Analysis in Psychology and Education. McGraw-Hill Education. New York.

Frixione, M. (2001),' Tractable competence'. Minds and Machines 11:379-97.

Gabbay, D. M. \& Moravcsik, J. M. E. (1974), 'Branching quantifiers, English and Montague grammar'. Theoretical Linguistics 1:140-57.

Garey, M. R. \& Johnson, D. S. (1979), Computers and Intractability. W. $\mathrm{H}$. Freeman and Co. San Francisco.

Geurts, B. \& van der Slik, F. (2005), 'Monotonicity and processing load'. Journal of Semantics 22:97-117.

Gierasimczuk, N. \& Szymanik, J. (2007), 'Hintikka's thesis revisited'. The Bulletin of Symbolic Logic 17:273.

Guenthner, F. \& Hoepelman, J. P. (1976), 'A note on the representation of branching quantifiers'. Theoretical Linguistics 3:285-89.

Heim, I., Lasnik, H., \& May, R. (1991), 'Reciprocity and plurality'. Linguistic Inquiry 22:63-101.

Henkin, L. (1961), 'Some remarks on infinitely long formulas'. In Infinistic Methods. Pergamon Press. Warsaw. 167-83.

Hintikka, J. (1973), 'Quantifiers vs. quantification theory'. Dialectica 27: 329-58.

Hintikka, J. (1976), 'Partially ordered quantifiers vs. partially ordered ideas'. Dialectica 30:89-99.

Immerman, N. (1998), Descriptive Complexity. Springer. New York.

Jackendoff, R. (1972), Semantic Interpretation and Generative Grammar. MIT Press. Cambridge, MA.
Janssen, T. (2003), 'On the semantics of branching quantifier sentences'. In P. Dekker \& R. van Rooij (eds.), Proceedings 14th Amsterdam Colloquium. University of Amsterdam. 147-51.

Jaszczolt, K. (2002), Semantics and Pragmatics: Meaning in Language and Discourse. Longman. London.

Kempson, R. M. \& Cormack, A. (1981a), 'Ambiguity and quantification'. Linguistics and Philosophy 4:259309.

Kempson, R. M. \& Cormack, A. (1981b), 'On 'formal games and forms for games". Linguistics and Philosophy 4: 431-35.

Kempson, R. M. \& Cormack, A. (1982), 'Quantification and pragmatics'. Linguistics and Philosophy 4:607-18.

Krynicki, M. \& Mostowski, M. (1995), 'Henkin quantifiers'. In M. Krynicki, M. Mostowski, \& L. Szczerba (eds.), Quantifiers: Logics, Models and Computation. Kluwer Academic Publishers. 193-263.

Liu, F.-H. (1996), 'Branching quantification and scope independence'. In J. van der Does \& J. van Eijck (eds.), Quantifiers, Logic and Language, Center for the Study of Language and Information. Standford. 155-68.

Lønning, J. T. (1997), 'Plurals and collectivity'. In J. van Benthem \& A. ter Meulen (eds.), Handbook of Logic and Language. Elsevier. The Netherlands. 1009-53.

May, R. (1985), Logical Form: Its Structure and Derivation. The MIT Press. Cambridge.

May, R. (1989), 'Interpreting logical form'. Linguistics and Philosophy 12:387-435.

Mostowski, M. (1994), 'Kwantyfikatory rozgalęzione a problem formy logicznej'. In M. Omyła (ed.), Nauka $i$ jezyk, Biblioteka Myśli Semiotycznej. Warsaw. 201-42. 
Mostowski, M. \& Wojtyniak, D. (2004), 'Computational complexity of the semantics of some natural language constructions'. Annals of Pure and Applied Logic 127:219-27.

Papadimitriou, C. H. (1993), Computational Complexity. Addison Wesley. California.

Robaldo, L. (2009), 'Independent set readings and generalized quantifiers'. Journal of Philosophical Logic, forthcoming.

Schlenker, P. (2006), 'Scopal independence: a note on branching and wide scope readings of indefinites and disjunctions'. Journal of Semantics 23:281-314.

Sevenster, M. (2006), Branches of Imperfect Information: Logic, Games, and Computation. Ph.D. thesis, Universiteit van Amsterdam. Amsterdam.

Sher, G. (1990), 'Ways of branching quantifiers'. Linguistics and Philosophy 13:393-442.
Soja, N., Carey, S., \& Spelke, E. (1991), 'Ontological categories guide young children's induction of word meaning: Object terms and substance terms'. Cognition 38:179-211.

Stenius, E. (1976), 'Comments on Jaakko Hintikka's paper "Quantifiers vs. quantification theory". Dialectica 30:67-88.

Szymanik, J. (2005), 'Problemy z formą logiczną. Studia Semiotyczne 25:187200.

Szymanik, J. (2009), Quantifiers in TIME and SPACE. Computational Complexity of Generalized Quantifiers in Natural Language. Ph.D. thesis, Universiteit van Amsterdam. Amsterdam.

Tennant, N. (1981), 'Formal games and forms for games'. Linguistics and Philosophy 4:311-20.

First version received: 25.8.2008 Second version received: 16.1.2009 Accepted: 25.4.2009 